\title{
Precision cancer therapy by molecular profiling - A Theoretical Study
}

\author{
V. Sah ${ }^{1}$ \\ ${ }^{1}$ SDIDTS
}

\begin{abstract}
The amount of druggable tumor-specific molecular aberrations has increased significantly over the last decade, with biomarker-matched therapies demonstrating a major survival advantage in many cancer forms. Therefore, molecular pathology has been critical not just for tumor detection and prognosis, but also for clinical decision-making in everyday practice. The advent of next-generation sequencing technology and the proliferation of large-scale tumor molecular profiling services through universities worldwide have transformed the area of precision oncology. When systematic genomic studies become more accessible in clinical and laboratory environments, healthcare professionals face the difficult challenge of outcome analysis and translation. This study summarizes existing and future methods to implementing precision cancer medicine, outlining the obstacles and possible strategies for facilitating the understanding and maximization of molecular profiling findings. Beyond tumor DNA sequencing, we discuss innovative molecular characterization techniques such as transcriptomics, immunophenotyping, epigenetic profiling, and single-cell analysis. Additionally, we discuss present and future uses of liquid biopsies for evaluating blood-based biomarkers such as circulating tumor cells and nucleic acids. Finally, the shortcomings of genotype-based treatments give insight into opportunities to extend personalized medicine beyond genomics.
\end{abstract}

Keywords: Tumor, Genomic profiling, cancer, genome.

\section{INTRODUCTION}

The world of oncology has seen dramatic improvements in the way cancer patients are treated during the last decade, including a move away from a "one-size-fits-all" paradigm to an increased emphasis on personalized medicine focused on genomic variants. Precision medicine in cancer is described as "the use of therapeutics that are intended to favor a subset of patients whose cancer exhibits particular molecular or cellular characteristics (most often genomic changes and changes in gene or protein expression patterns)" [1]. In some cancers, molecular profiling can often include diagnostic and prognostic knowledge that is clinically important. Precision medicine has been facilitated by an expanding body of information identifying key drivers of oncogenesis, advancements in tumor analysis through next-generation sequencing (NGS) and other profiling technologies, and the availability of new therapeutic agents. Precision medicine has now improved cancer care: tailored treatments will already be used to optimize health conditions in patients with both common and unusual malignancies (Table 1). This study examines existing and evolving practices, addresses achievements and obstacles, and suggests possible alternatives for precision medicine adoption in clinical science and practice (Fig. 1). The extension of precision medicine's therapeutic effectiveness and reach by incorporating additional molecular characterization tools outside genomics, such as transcriptomics, epigenetics, and immunophenotyping, as well as by evaluating medication formulations in addition to monotherapy approaches. Finally, since patients are willing participants in precision medicine programs, money must be allocated to maximize their knowledge and participation.

\section{ADVANCES IN MOLECULAR TECHNIQUES HELP PRECISION MEDICINE}

The analysis and quality of treatment testing relationships must be emphasized above all others. Large-scale study trials can discover new biomarkers of clinical value, which can then be taken up as a new standard of treatment. Prevalent molecular modifications, such as protein overexpression, mutations in driver genes, or rearrangements, are well-established predictive biomarkers of reaction to targeted therapies. Molecular pathology research has become an important laboratory method for characterizing tumor biology and for guiding therapeutic decisions. 
Immunohistochemistry (IHC) and fluorescence in situ hybridization (FISH) are basic precision medicine instruments, with many biomarkers already observed by these two techniques (Table 1). They are able to diagnose all gene aberrations (e.g. gene amplifications) and complex DNA rearrangements or point mutations (such as EML4ALK translocation in non-small cell lung cancer (NSCLC) and IDH1/2 mutations in glioma). An additional application of IHC has been used in PD-L1 and mismatch repair status. This allows the use of IO-based treatments including anti-PD-1/PD-L1 agents in some tumor forms [1, 4, 5, 8-10]. FISH has become the gold standard for measuring DNA rearrangements, and it is often routinely used to prove gene amplifications.

Increasingly, NGS innovations are supplanting traditional approaches, such as single-gene research, and targeted mutational platforms in routine molecular pathology. High sensitivity and specificity are commonly achieved in determining actionable mutations that either match selected therapies or are seen to cause resistance to particular drugs [13-16]. However, in order to be identified, a purpose-made assay is needed for each biomarker. The simultaneous study of mutations, copy number variations (CNV), translocations, and fusions in several genes is possible via NGS. As opposed to serial single-biomarker analyses, genomic complexity necessitates more effective, cost- and tissue-saving tumor analyses, especially in the sense of that complexity. Similar sensitivity and specificity were seen in several studies that compare NGS success against RT-PCR-based targeted mutation platforms, singlegene monitoring, and other traditional techniques.

In the sense of precision cancer medicine, moving to systematic genomic profiling by NGS offers significant advantages, including reduced costs and shortened processing time.

\section{A. Current NGS approaches: gene panels versus entire exome and whole genome sequencing}

Targets may be pre-specified gene panels, concentrate on the coding regions of any of the base pairs of the genome (exome sequencing), or consider the whole tumor genome, including intronic regions (whole genome sequencing). the difference between the clinical and laboratory tests is determined by many variables (Additional file 1) 17 WES and WGS have been in the science field with the aim of gathering vast volumes of genomic information to advance translational studies for the betterment of cancer research. Targeter gene panels have been utilized in clinical settings, as they offer increased coverage of hotspot regions with identified actionable mutations, quicker turnaround, and more clinically applicable results. The number of genes used in these panels ranges from 20 to over 400. Despite the uncertainty about the therapeutic usefulness of evaluating all of the genes that are found in vast panels, the expense of including new genes is a motivator for doing more rigorous genomic profiling in the clinic.

After finding $40-50 \%$ of patients to have actionable defects in their driver genes, a slightly smaller percentage of patients (10-15\%) receive the genotypematched medications [23-28]. The main issue is the shortage of authorised or investigational agents that target individual driver alterations. Recruiting people with unusual mutations for clinical trials may be difficult, which slows down the discovery of experimental medicines. In addition, the variability of intratumoral mutations, which is the trunk vs branch mutations, and the presence of a specific molecular alteration can influence the treatment In this respect, [30] has recently discovered that several somatic hotspot mutations that are

and ALK gene research, with panel testing providing new possibilities for selective treatment to less than 5\% of patients However, a broader genomic analysis, which looks beyond actionable variants in known genes, has the potential to include information on acquired resistance to treatments (such as the EGFR T790M mutation) or to propose novel drug combinations (such as downstream activation of the BRAF pathway leading to the combination of BRAF and MEK inhibitors in BRAF mutant melanoma). The previously reported results allowed for the incorporation of new therapeutic strategies in patients with EGFR-mutant and BRAF-mutant NSCLC (such as osimertinib) [34, 35] and melanoma (such as the dabrafenib and trametinib combination) [30], resulting in substantial improvements in survival.

$\mathrm{SNV}, \mathrm{CNV}$, and molecular rearrangements such as gene fusions may be detected simultaneously in tumor samples using more advanced sequencing technologies such as WGS. One latest research characterized 2500 samples of metastatic tumors with paired normal tissue. As the result of WGS, the list of genetic variants contained in each Pediatric pan-cancer cohorts were often used to classify the causal genes. New sequencing methods will now identify deep structural changes in the genome for more than a few kilobases [24].

SNV, CNV, and methylation profiles were detected simultaneously by a low-pass WGS technique utilizing longread sequencing. Most RNA sequencing attempts have been aimed at discovering new fusion and splicing isoforms important to tumor development or drug resistance [24-25]. Despite the negative error rates for long-read technologies, somatic variant detection is still in its early stages of growth. 


\section{GENOMIC FINDINGS}

The effect of tumor variants on particular patients' health is an active area of research in precision cancer medicine [13]. Genomic databases and web tools have collected associations with a particular histology or illness, as well as their prognostic and/or predictive importance in exposure to specific therapies [17]. through translational research applications (e.g., The Cancer Genome Atlas (TCGA), International Cancer Genome Consortium (ICGC)) to the most current data on the clinical benefits obtained from matched drugs that can be used to drive variantspecific treatment recommendations (e.g., OncoKB, MyCancerGenome, CIViC) Many other reports depend on passenger variant curation (dbCPM). Data sets such as MyCancerGenome or CIViC also help to discriminate driver variants (which are involved in tumor development and proliferation). In view of the recent observations from Hess et al. [20], these datasets will need to be revisited. at the gene stage, current statistical models are flawed and could contribute to high rates of false positives

i.e., identifying passenger variants which are, in reality, drivers. The proposed new model by Hess et al. [20] allows for mutability on the basis of single base pairs, increasing both strength and precision. For novel variants that have not previously been published, some website-based approaches can help identify possible biological effects on the basis of in silico tools and metaprediction methods (e.g., dbNSFP). ClinVar, OMIM, and other germline-based genomic databases (e.g., NCBI ClinVar) that compile previously documented germline polymorphisms can also help in differentiating tumor-specific somatic variants from non-pathogenic DNA variations in patients for whom no matched normal tissue or blood is accessible [12, 13]

Getting many knowledgebases brings with it the drawback of scattering genomic material.

Tools that make user-friendly disorder, genome, variant, and drug integration possible, with the aim of assisting clinical interpretation have been created [14]. In addition, various platforms and curators may contribute to inaccurate or incomplete data collection on variants, which results in different format presentation and terminology, thereby hindering the ability to view variant actionability. Further work is needed to guarantee the usefulness of genomic data. It is recommended that minimal variant level data (MVLD) be curated with an emphasis on clinical usefulness by the Somatic Working Group of the Clinical Genome [14]. The genomic descriptive knowledge often includes information on the clinical effects, such as biomarker class (diagnostic, prognostic, predictive), matched medications accessible, and therapeutic effect (responsive vs resistant). Also, the Global Alliance for Genomics Health (GA4GH) Variant Interpretation for Cancer Consortium (VICC) have been suggested [15].

Standardizing somatic variant description, labeling, and documentation is essential for a variety of agencies and practitioners to use. a collection of guidelines for the classification and reporting of somatic variants in cancer patients has been issued by the American Society of Clinical Oncology, the Association for Molecular Pathology, and the College of American Pathologists [16]. Evidence-based variant categorization is useful in facilitating clinical decision-making.

Gene mutations in tumor profiling patients have been identified $[16,17]$. In cancer patients with a high likelihood of having a genetic predisposition, this is important. Colorectal cancer surveillance for intermittent vs hereditary Lynch syndrome, for example, typically involves a multi-step procedure that uses sequential IHC, microsatellite instability, and additional molecular testing for somatic modifications. sequencing for mismatch repair proteins, sequencing for other repeated somatic modifications (e.g., BRAF), and evaluation of microsatellite instability demonstrated greater flexibility in detecting Lynch syndrome in patients with colorectal cancer for inherited germline mutations in BRCA1 and BRCA2, somatic tumor profiling of PARP inhibitor may show. Germlinefocused research should be conducted during tumor-only genomic profiling to distinguish variants with a large allele frequency (> $20 \%$ to $30 \%$ ) and selected genes of clinical significance (27). Long-term follow-up can also benefit from genetic subspecialties.

\section{CONCLUSIONS}

Precision medicine, which uses molecular profiling tools to improve detection, prognosis, and analysis of health results, is rapidly being combined with traditional clinicopathological assessments. In multiple fronts, NGS and other omics-based experiments remain yet to be proved useful. an image of the future of precision oncology will integrate comprehensive multi-omic tumor characterization, dynamic liquid biopsy monitoring, annotation that is automated by artificial intelligence but driven by clinicians, and enrollment of patients into innovative clinical trials that not only test molecular profile-drug matching but also investigate the utility of different drug-assignment algorithms [17] It would be necessary to not only apply genomics beyond genomics from a scientific standpoint, but also expand access to genotype-drug matching and create novel and flexible clinical trial designs to allow patients and clinicians to obtain more data. 


\section{REFERENCES}

[1] R. Patel, A. Kunche, N. Mishra, Z. Bhai yat, R. Joshi, Comparative review of existing mobile payment systems, International Journal of Applied Engineering Research, 10 (2015), pp. 16873-16884

[2] Bowman, M., Debray, S. K., and Peterson, L. L. 1993. Reasoning about naming systems.

[3] Ding, W. and Marchionini, G. 1997 A Study on Video Browsing Strategies. Technical Report. University of Maryland at College Park.

[4] Fröhlich, B. and Plate, J. 2000. The cubic mouse: a new device for three-dimensional input. In Proceedings of the SIGCHI Conference on Human Factors in Computing Systems

[5] Tavel, P. 2007 Modeling and Simulation Design. AK Peters Ltd.

[6] Stott JRR. Orientation and disorientation in aviation. Extreme Physiology \& Medicine. 2013; 2: 2.doi: 10.1186/2046-7648-2-2?optIn=true.

[7] Newman RL, Rupert AH. The magnitude of the spatial disorientation problem in transport airplanes. Aerosp Med Hum Perf. 2020; 91(2): 65-70.doi: 10.3357/AMHP.5442.2020.

[8] Gillingham KK. A primer of vestibular function, spatial disorientation, and motion sickness. Aeromedical Reviews. 1966; 4: 1-80.

[9] Upton K, Modi A, Patel K, et al. Epigenomic profiling of neuroblastoma cell lines. Sci Data. 2020;7(1):116. Published 2020 Apr 14. doi:10.1038/s41597-020-0458-y

[10] Gillingham KK. The spatial disorientation problem in the United States Air Force. Journal of vestibular research: equilibrium \& orientation. 1992; 2(4): 297-306.

[11] Lawson B, McGrath B, Rupert A, Thompson LI, Brill JC, Kell AM. A countermeasure for loss of situation awareness: Transitioning from the laboratory to the aircraft. 2016 IEEE Aerospace Conference, Big Sky, USA, 2016

[12] Kendsersky NM, Lindsay J, Kolb EA, et al. The B7-H3-Targeting Antibody-Drug Conjugate m276-SL-PBD Is Potently Effective Against Pediatric Cancer Preclinical Solid Tumor Models. Clin Cancer Res. 2021;27(10):2938-2946. doi:10.1158/1078-0432.CCR-20-4221

[13] Shiff NJ, Oen K, Rabbani R, Lix LM. Validation of administrative case ascertainment algorithms for chronic childhood arthritis in Manitoba, Canada. Rheumatol Int. 2017;37(9):1575-1584. doi:10.1007/s00296-017$3734-1$

[14] Raman, Swetha and Buongervino, Samantha N. and Lane, Maria and Zhelev, Doncho V. and Zhu, Zhongyu and Cui, Hong and Martinez, Benjamin and Martinez, Daniel and Wang, Yanping and Upton, Kristen A. and Patel, Khushbu and Rathi, Komal S. and Navia, Carmen T. and Harmon, Daniel B. and Pawel, Bruce and Dimitrov, Dimiter and Maris, John M. and Julien, Jean-Philippe and Bosse, Kristopher, The D3-GPC2-PBD ADC is Potently Efficacious Against Neuroblastoma and SCLC Via Engagement of a Conformational GPC2 Epitope (September 2020). Available at SSRN: https://ssrn.com/abstract=3673604 or http://dx.doi.org/10.2139/ssrn.3673604

[15] Stuart S, Hickey A, Vitorio R, et al. Eye-tracker algorithms to detect saccades during static and dynamic tasks: a structured review. Physiol Meas. 2019;40(2):02TR01. Published 2019 Feb 26. doi:10.1088/13616579/ab02ab

[16] Patel, R., Kunche, A., Mishra, N., Bhaiyat, Z., \& Joshi, P. R. (2015). Paytooth - a cashless mobile payment system based on Bluetooth. International Journal of Computer Applications, 120, 3843. https://doi.org/10.5120/21412-4450. 
[17] Patel, Rushabh. "Predicting Invasive Ductal Carcinoma Using a Reinforcement Sample Learning Strategy Using Deep Learning.” ArXiv:2105.12564 [Cs, Eess], May 2021. arXiv.org, http://arxiv.org/abs/2105.12564.

[18] Rokita JL, Rathi KS, Cardenas MF, et al. Genomic Profiling of Childhood Tumor Patient-Derived Xenograft Models to Enable Rational Clinical Trial Design. Cell Rep. 2019;29(6):1675-1689.e9.

doi:10.1016/j.celrep.2019.09.071

[19] Upton K, Modi A, Patel K, et al. Epigenomic profiling of neuroblastoma cell lines. Sci Data. 2020;7(1): 116. Published 2020 Apr 14. doi:10.1038/s41597-020-0458-y

[20] Mahajan V, Venugopal VK, Murugavel M, Mahajan H. The Algorithmic Audit: Working with Vendors to Validate Radiology-AI Algorithms-How We Do It. Acad Radiol. 2020;27(1):132-135. doi:10.1016/j.acra.2019.09.009

[21] Buxton, E. K., Vohra, S., Guo, Y., Fogleman, A., \& Patel, R. (2019). Pediatric population health analysis of southern and central Illinois region: A cross sectional retrospective study using association rule mining and multiple logistic regression. Computer methods and programs in biomedicine, 178, 145-153.

[22] Campbell E. Random Compiler for Fast Hamiltonian Simulation. Phys Rev Lett. 2019;123(7):070503. doi:10.1103/PhysRevLett.123.070503

[23] Devkota S, Aschwanden P, Kunen A, Legendre M, Isaacs KE. CcNav: Understanding Compiler Optimizations in Binary Code. IEEE Trans Vis Comput Graph. 2021;27(2):667-677. doi:10.1109/TVCG.2020.3030357

[24] Rushabh Patel and Rahul Raghvendra Joshi, Envision of I-RS (I-Railway System) - based on Cloud Computing, International Journal of Science Engineering and Technology Research (IJSETR), vol. 4, no. 1, January 2015.

[25] Yang X, He H. An advanced compiler designed for a VLIW DSP for sensors-based systems. Sensors (Basel). 2012;12(4):4466-4478. doi:10.3390/s120404466

[26] Rushabh Patel and Rahul Raghvendra Joshi, Introducing a Hi-Tech - Cloud based Public Transport System, International Journal of Computational Intelligence Research ISSN 0973-1873 Volume 11, Number 1 (2015), pp. 19-26

[27] Minkovich K, Srinivasa N, Cruz-Albrecht JM, Cho Y, Nogin A. Programming time-multiplexed reconfigurable hardware using a scalable neuromorphic compiler. IEEE Trans Neural Netw Learn Syst. 2012;23(6):889-901. doi:10.1109/TNNLS.2012.2191795

[28] Rushabh Patel and Rahul Raghvendra Joshi, Evolution \& Envisage of Mobile Network, International Journal of Scientific Engineering and Technology Research ISSN 2319-8885 Vol.03, Issue.01 (January-2014), pp. 0136-0143.

[29] Patel, Rushabh. "Predicting Invasive Ductal Carcinoma Using a Reinforcement Sample Learning Strategy Using Deep Learning.” ArXiv:2105.12564 [Cs, Eess], May 2021. arXiv.org, http://arxiv.org/abs/2105.12564.

[30] Patel, Rushabh, and Yanhui Guo. "Graph Based Link Prediction between Human Phenotypes and Genes.” ArXiv:2105.11989 [Cs], May 2021. arXiv.org, http://arxiv.org/abs/2105.11989.

[31] Lucas J. Vespa et al, A Novel Reinforcement Sample Learning Strategy for Convolution Neural Network in Computer Aided Diagnosis System for Breast Cancer, SIIM 2017 Scientific Session, Posters \& Demonstrations

[32] Lunter G. HMMoC--a compiler for hidden Markov models. Bioinformatics. 2007;23(18):2485-2487. doi:10.1093/bioinformatics/btm350 